\title{
Experiência no tratamento de aneurismas cerebrais após a implantação da embolização
}

\author{
Daniel Rodrigues de Oliveira1, Pedro Pianca Neto¹, Emerson Sena', \\ José Carlos Mariano Viana de Souza', Bernardo de Andrada', \\ Werther Garfield de Almeida², Francisco Doutel ${ }^{3}$, Armando da Glória Júnior ${ }^{3}$, \\ Francisco Weldes ${ }^{3}$, Wagner Mariushi', José Renato Ludolf Paixão ${ }^{3}$, Carlos Henrique Ribeiro ${ }^{5}$
}

Serviço de Neurocirurgia Prof. Oscar Fontenelle do Hospital Municipal Salgado Filho (HMSF), Rio de Janeiro, RJ.

\section{RESUMO}

Objetivo: Demonstrar os resultados do Serviço comparando embolização e clipagem no tratamento dos aneurismas intracranianos durante o período de 1/9/2007 a 1/12/2008, quando a técnica de embolização foi implantada no Serviço. Pacientes e métodos: Foi realizado estudo retrospectivo no banco de dados do hospital com programa estatístico (Epi Info 2000 ${ }^{\circledR}$ e colhidas informações mediante análise do registro cirúrgico no Serviço naquele período. Os dados foram cruzados pelos dois métodos para cálculo de parâmetros estatísticos. Resultados: No período, foram tratados 61 pacientes: 43 mulheres e 18 homens; em 40 o tratamento foi a embolização e em 21, a clipagem. O tempo médio de permanência dos pacientes submetidos à clipagem foi de 37 dias e o dos submetidos à embolização, de 18,5 dias. Dos 21 pacientes do primeiro grupo, 47\% apresentavam idade entre 51 e 60 anos e dos 40 do grupo da embolização, 55\% tinham idade entre 41 e 60 anos. A mortalidade global foi de 11,47\%; 9,5\% nos casos de clipagem e 12,5\% no grupo da embolização. Conclusão: Observou-se que a embolização reduziu o tempo médio de permanência e, indiretamente, custos hospitalares, não alterando a taxa de mortalidade. A maioria dos aneurismas diagnosticados era roto, justificando o grande período de internação e a taxa de óbitos. O emprego da embolização foi uma conquista importante do Serviço para a rede municipal do Rio de Janeiro na terapêutica dos aneurismas.

PALAVRAS-CHAVE

Aneurisma intracraniano. Embolização. Clipagem.

\section{ABSTRACT}

Experience after embolization technique standard use in the treatment of cerebral aneurysms Objective: To demonstrate the results of comparative study between surgical approaches versus embolization in the treatment of cerebral aneurysms in our Service. Patients and methods: Retrospective analysis using the hospital's databank, with statistical software (Epi Info $2000^{\circledR}$ ). The period of study was September 1, 2007 to December 1, 2008. The data was crossed using statistics parameters. Results: In that period 61 patients were collected, 43 females and 18 males; 40 patients received the treatment by embolization and 21 were submitted to surgery (aneurismal clipping). The hospitalization overall time was 37 days for open surgery and 18.5 days when embolization was the choice. Among open surgery group, $47 \%$ aged 51 to 60 years and among the embolization group 55\% aged 41 to 60 years. The global mortality was $11.47 \%$; $9.5 \%$ in aneurismal clipping and $12.5 \%$ in embolization. Conclusion: The embolization presented shorter hospitalization time with lower hospitals coasts. Most of the aneurysms had ruptured, explaining the long hospitalization time; besides the subarachnoid hemorrhage co-morbities had significative contribution in the mortality. The use of embolization in neurosurgery was offers an advance for the intracranial aneurysms. The possibility of choice between the two methods was an important conquest in public health Service of Rio de Janeiro city.

\section{KEY WORDS}

Intracranial aneurysms. Embolization. Clipping.

1. Residente de Neurocirurgia do Hospital Municipal Salgado Filho (HMSF).

2. Médico chefe da Epidemiologia do HMSF.

3. Neurocirurgião estatutário HMSF.

4. Neurocirurgião e neurorradiologista membro da Sociedade Brasileira de Neurocirurgia (SBN).

5. Neurocirugião, chefe do Serviço de Neurocirurgia do HMSF, 


\section{Introdução}

A técnica de embolização de aneurismas intracranianos foi introduzida em 1991 e atualmente é uma alternativa menos invasiva para o tratamento dos aneurismas intracranianos, tendo sido aprovada pelo FDA em 1995. ${ }^{1,4,5}$

O nosso hospital é um centro de referência da rede municipal do Rio de Janeiro no tratamento das doenças vasculares cerebrais, o único da rede com alta complexidade para a especialidade de neurocirurgia, possui 323 leitos gerais e 30 leitos de neurocirurgia, $8 \%$ do total. De janeiro de 2006 a outubro de 2008, o Serviço realizou 10.561 internações e 1.997 cirurgias, com 61 aneurismas clipados no período; em 2007 foram registradas 1.053 internações e realizadas 770 neurocirurgias e em 2008, até outubro, 860 internações com 580 neurocirurgias.

O Serviço passou a realizar o tratamento endovascular para aneurismas intracranianos oficialmente em setembro de 2007.

O presente artigo pretende demonstrar a experiência do Serviço e os resultados após a implementação da embolização como opção de tratamento menos invasivo e com menores morbidades para os pacientes com aneurismas intracranianos, além do tratamento tradicional, que é a clipagem cirúrgica.

\section{Materiais e métodos}

As informações foram coletadas do banco de dados do hospital, no Serviço de Epidemiologia, por meio do programa Epi Info $2000^{\circledR}$, durante o período de $1 / 9 / 2007$ a 1/12/2008, no qual se passou a realizar o tratamento por meio da técnica de embolização. A coleta dos dados foi realizada de forma retrospectiva. Foram analisados apenas os pacientes que se submeteram ao tratamento do aneurisma, um total de 61; desses, 21 foram submetidos à clipagem cirúrgica $(34,4 \%)$ e 40 , embolizados $(65,6 \%)$.

De rotina, todos os pacientes que adentram na emergência são submetidos à tomografia computadorizada de crânio para diagnóstico da hemorragia subaracnoidea ou punção lombar e, quando pertinente, é realizada arteriografia cerebral dos quatro vasos, mediante a técnica de Seldinger. Esse exame é realizado com agendamento via atendimento ambulatorial para os pacientes com encaminhamento. O exame é discutido em seção clínica e, então, é escolhido o método de tratamento.

A análise das variantes foi cruzada por: sexo, idade, tipo de alta, taxa de óbito, sobrevida, tempo médio e global de internação e, então, comparados os dois métodos.

\section{Resultados}

Dos 21 pacientes submetidos à clipagem dos aneurismas, 4 eram homens e 17, mulheres. A distribuição etária está apresentada na tabela 1 . O tempo médio de permanência hospitalar foi de 37 dias, mínimo de 14 dias e máximo 59 dias, com mediana de 35,5 dias e moda de 31 dias (Tabela 2).

Foram realizadas 29 cirurgias, que incluíram: realização de derivações ventriculares, drenagem de hematomas como complicações do sangramento e ressangramento. Total de dois óbitos, ou 9,5\% (Tabela 2).

Dentre os 40 pacientes submetidos à embolização dos aneurismas, 26 eram mulheres e 14, homens. A distribuição etária está apresentada na tabela 3. O tempo médio de permanência hospitalar foi de 18,5 dias com permanência mínima de 2 dias e máxima de 47 dias, com mediana de 17,3 dias e moda de 21 dias (Tabela 4).

Foram realizados 60 procedimentos nesse grupo, que incluíram, além da embolização, derivações ventriculares, drenagem de hematomas como complicações do sangramento. Ocorreu óbito em cinco, com taxa de mortalidade de $12,5 \%$ (Tabela 4 ).

\begin{tabular}{|c|c|c|c|c|}
\hline \multicolumn{5}{|c|}{$\begin{array}{c}\text { Tabela } 1 \\
\text { Distribuição etária e gênero - } 21 \text { casos clipagem }\end{array}$} \\
\hline Idade (anos) & Homens & \multicolumn{2}{|c|}{ Mulheres } & Totais \\
\hline $31-40$ & 00 & \multicolumn{2}{|c|}{02} & 02 \\
\hline $41-50$ & 01 & \multicolumn{2}{|c|}{05} & 06 \\
\hline $51-60$ & 03 & \multicolumn{2}{|c|}{08} & 11 \\
\hline $61-70$ & 00 & \multicolumn{2}{|c|}{02} & 02 \\
\hline Totais & 04 & \multicolumn{2}{|c|}{17} & 21 \\
\hline \multicolumn{5}{|c|}{$\begin{array}{l}\text { Tabela } 2 \\
\text { evolutiva }\end{array}$} \\
\hline Idade (anos) & Sobrevida & Óbitos & Totais & TMP $^{*}$ \\
\hline $31-40$ & 01 & 01 & 02 & 39,5 \\
\hline $41-50$ & 06 & 00 & 06 & 38,2 \\
\hline $51-60$ & 11 & 00 & 11 & 36,2 \\
\hline $61-70 \mathrm{a}$ & 01 & 01 & 02 & 33,0 \\
\hline Totais & 19 & 02 & 21 & $36,5^{* *}$ \\
\hline
\end{tabular}

(") TMP: Tempo médio de permanência hospitalar, em dias. (") Média de TMP.

\begin{tabular}{lccc}
\hline \multicolumn{5}{c}{ Tabela 3} \\
\multicolumn{2}{c}{ Distribuição } & etária & e \\
gênero & $-\mathbf{4 0}$ casos embolizados \\
\hline Idade (anos) & Homens & Mulheres & Totais \\
$11-20$ & 01 & 00 & 01 \\
$21-30$ & 00 & 02 & 02 \\
$31-40$ & 06 & 04 & 10 \\
$41-50$ & 02 & 11 & 13 \\
$51-60$ & 04 & 07 & 11 \\
$61-70$ & 01 & 02 & 03 \\
Totais & 14 & 26 & 40 \\
\hline
\end{tabular}


Tabela 4

Distribuição etária e evolutiva - 40 casos embolizados

\begin{tabular}{lcccc}
\hline Idade (anos) & Sobrevida & Óbitos & Totais & TMP $^{*}$ \\
$11-20$ & 01 & 00 & 01 & 13,0 \\
$21-30$ & 02 & 00 & 02 & 22,5 \\
$31-40$ & 10 & 00 & 10 & 18,4 \\
$41-50$ & 12 & 01 & 13 & 20,4 \\
$51-60$ & 07 & 04 & 11 & 17,4 \\
$61-70$ & 03 & 00 & 03 & 12,3 \\
Totais & 35 & 05 & 40 & $17,3^{* * *}$ \\
\hline
\end{tabular}

(") TMP: Tempo médio de permanência hospitalar, em dias. (") Média do TMP.

Para os dois grupos, a taxa de mortalidade geral foi de $11,5 \%$. A redução importante do tempo de permanência daqueles submetidos à embolização quando comparada com aqueles submetidos à clipagem cirúrgica é demonstrada nas tabelas 5 e 6 .

\begin{tabular}{lcccc}
\hline \multicolumn{5}{c}{ Tabela 5 } \\
Comparativo etário & e & TMP \\
Embolizados & Embolizados $\times$ clipagem \\
\hline Idade (anos) & Totais & TMP & Totais & TMP \\
$11-20$ & 01 & 13,0 & 00 & 00,0 \\
$21-30$ & 02 & 22,5 & 00 & 00,0 \\
$31-40$ & 10 & 18,4 & 02 & 39,5 \\
$41-50$ & 13 & 20,4 & 06 & 38,3 \\
$51-60$ & 11 & 17,4 & 11 & 36,2 \\
$61-70$ & 03 & 12,3 & 02 & 33,0 \\
Totais & 40 & $17,3^{* *}$ & 21 & $24,5^{* *}$ \\
\hline
\end{tabular}

(") TMP: Tempo médio de permanência hospitalar, em dias. ("*) Média do TMP.

\begin{tabular}{|c|c|c|c|}
\hline \multicolumn{4}{|c|}{$\begin{array}{c}\text { Tabela } 6 \\
\text { Comparativo global do } \text { TMP }^{\star}-\text { Embolizados } \times \text { clipagem }\end{array}$} \\
\hline Idade (anos) & Embolizados & Clipagem & Global \\
\hline $11-20$ & 13,0 & 00,0 & 06,5 \\
\hline $21-30$ & 22,5 & 00,0 & 11,2 \\
\hline $31-40$ & 18,4 & 39,5 & 28,9 \\
\hline $41-50$ & 20,4 & 38,3 & 29,3 \\
\hline $51-60$ & 17,4 & 36,2 & 26,8 \\
\hline $61-70$ & 12,3 & 33,0 & 22,6 \\
\hline Totais & $17,3^{* *}$ & $24,5^{* *}$ & $20,9^{* *}$ \\
\hline
\end{tabular}

(") TMP: Tempo médio de permanência hospitalar, em dias. ("*) Média do TMP.

\section{Discussão}

O tratamento endovascular para aneurismas intracranianos é uma técnica relativamente nova, tendo sido introduzido, em 1991, o Guiglielmi detachable coil (Boston Scientific/Target, Fremont, CA) como alternativa à clipagem cirúrgica. Foi inicialmente usado para investigação clínica em 1991 e aprovado pelo FDA em 1995. Desde então, o tratamento endovascular tem ganhado a preferência para o tratamento de determinados tipos e anatomias de aneurismas intracranianos, com relativa facilidade de acesso e invasão mínima, principalmente ao sistema vertebrobasilar. ${ }^{1,4}$

Essa técnica foi introduzida na rede municipal do Rio de Janeiro por meio do nosso Serviço e permitiu o tratamento pelo SUS. A opção do tratamento endovascular sem a necessidade de transferência para outras unidades para a realização do procedimento facilita $o$ tratamento do paciente.

$\mathrm{O}$ volume de pacientes embolizados representou a maioria: 40 pacientes comparados com 21. Pelo fato de o procedimento endovascular ter uma logística mais facilitada no hospital, poder ser realizado com anestesia local, ser realizado logo após a arteriografia diagnóstica, com 24 horas de permanência no CTI e a alta em até 48 horas após o procedimento; o tempo mínimo de até dois dias de permanência hospitalar, entre outros fatores, influiu também no reduzido tempo médio e global de permanência hospitalar, que foi aproximadamente $50 \%$ menor comparado aos que foram submetidos a craniotomia e clipagem (Tabelas 5 e 6), reduzindo custos hospitalares, diárias em CTI, visitas médicas e custos com medicação.

Outro resultado que chamou a atenção foi a mortalidade de $10 \%$ para os submetidos à clipagem cirúrgica (Tabela 2) e de 12,8\% entre os submetidos à embolização (Tabela 4). Todos os pacientes que foram submetidos à craniotomia não foram operados na fase aguda; a cirurgia foi eletiva, ou seja, em suas melhores condições clínicas; a equipe possui neurocirurgiões vasculares experientes e uma equipe treinada com um excelente CTI pós-operatório. Alguns dos pacientes embolizados eram pacientes com escore IV na escala de Hunt-Hess, que já estavam em ambiente intensivo e não tinham condições de se submeter ao procedimento cirúrgico, ou seja, pacientes mais graves eram embolizados, por isso não há como comparar estatisticamente a mortalidade dos dois grupos, porque eles são compostos de pacientes diferentes.

Em sua grande maioria, o grupo de 61 pacientes foi composto de pacientes na fase aguda do sangramento do aneurisma, pelo fato de nosso hospital ter atendimento de emergência $24 \mathrm{~h}$, e com isso foram observadas complicações inerentes ao sangramento dos aneurismas como vasoespasmo, ressangramento, hidrocefalia etc.

\section{Conclusão}

Nosso objetivo com este trabalho não é demonstrar qual a melhor técnica para o tratamento dos aneurismas 
intracranianos ou qual apresenta os melhores resultados, como em outros estudos ${ }^{5}$, mas comprovar, mediante resultados, a importância de poder realizar ambos os procedimentos em nosso hospital geral, permitindo ao paciente ter a opção de escolher seu tratamento, seja pela clipagem ou embolização.

A realização da embolização na rede municipal, apesar de ser um procedimento que demanda alto investimento financeiro e com alto custo, demonstrou a redução importante de gastos hospitalares pela redução do tempo médio e global de internação em até 50\%, representados nas tabelas 5 e 6, assim como redução dos custos com diárias em CTI, entre outros.

Com a soma de todos esses fatores, concluímos que a realização dos dois métodos de tratamento foi uma conquista importante para a rede pública municipal do Rio de Janeiro. Em nosso hospital, a maioria dos aneurismas diagnosticados é rota, justificando o grande período de internação hospitalar devido à morbimortalidade do sangramento, contribuindo de forma significativa para a taxa de óbitos. O emprego da embolização na neurocirurgia foi um avanço na terapêutica dos aneurismas intracranianos.

Essa nova técnica serviu de grande experiência e crescimento profissional da equipe e para o Serviço de Neurocirurgia, assim como para os residentes. Logo após iniciarmos a embolização em larga escala, havia uma grande indicação para a embolização; com o tempo e adaptação, foi elaborada uma rotina para a indicação da melhor técnica de tratamento. Hoje seguimos no aprimoramento de ambas as técnicas tanto na modernização do material utilizado quanto na abrangência da área de atuação, tornando-nos referência para toda a rede municipal do Rio de Janeiro, recebendo pacientes de vários hospitais com doenças cerebrovasculares para a realização de diagnóstico e tratamento.
Como em toda rede pública do país, existem dificuldades de logística no hospital que, com o tempo, procuramos superar, para manter e aumentar o grau de qualidade e excelência que nosso Serviço representa para a cidade e para o estado do Rio de Janeiro.

\section{Referências}

1. Bairstow P, Dodgson A, Linto L, Khangure M. Comparison of cost and outcome of endovascular and neurosurgical procedures in the treatment of ruptured intracranial aneurism. Australas Radiol. 2002;46:249-51.

2. Ballet AC, Guérin J, Berge J, Taboulet F, Martin S, Philip V, et al. Traitment neurochirurgical et endovasculaire des anévrismes intracrâniens. Approche économique de deux alternatives thérape sur le $\mathrm{CHU}$ de Bordeaux. Neurochirurgie. 2002;48:419-25.

3. Molyneux AJ, Kerr R, Stratton I, Sandrecock P, Clarke M, Shrimpton J, et al. International Subarachnoid Aneurism Trial (ISAT) Collaborative Group: International Subaracnoid Aneurism Trial (ISAT) of neurosurgical clipping versus endovascular coiling in 2143 patients with ruptured intracranial aneurisms, a randomized trial. Lancet. 2002;360:1267-74

4. Pushpa VR, Huang J, Germanwala AV, Gailoud P, Murphy KP, Tamargo RJ. Microsurgical clipping and endovascular coiling of intracranial aneurisms: a critical review of the literature. Neurosurgery. 2008;62:1187-203.

5. Takao H, Nojo T, Ohtomo K. Treatment of ruptured intracranial aneurysms: a decision analysis. Brit $\mathrm{J}$ Radiol. 2008;81:299-303

Endereço para correspondência

Carlos Henrique Ribeiro

Hospital Municipal Salgado Filho, $7^{\circ}$ andar

Rua Arquias Cordeiro, 370

20770-000 - Rio de Janeiro, RJ, Brasil 